\title{
Hábitos saludables, motivos y barreras en la realización de actividad física en estudiantes universitarios*
}

\author{
Fredy Alexander Rodríguez Castellanos** \\ Sandra Carolina Valencia** \\ Erika María Gaitán Guzmán ${ }^{* * * *}$ \\ Sergio Andrés González Rodríguez ${ }^{* * * *}$ \\ Juan David León Díaz ${ }^{* * * * * *}$
}

Recibido: febrero 12 de 2017 • Evaluado: marzo 11 de 2017

Aceptado: marzo 17 de 2017

Resumen

El principal objetivo de la investigación fue conocer los motivos, barreras y hábitos saludables asociados a la actividad física en estudiantes de Psicología en una universidad de Bogotá. La muestra final fue de 145

" Proyecto de investigación desarrollado entre septiembre de 2014 y enero de 2017. Línea Calidad de Vida y Bienestar en Contextos de Salud. Grupo de Investigación Psicología, Salud, Trabajo y Calidad de Vida. Facultad de Psicología, Universidad Santo Tomás. Financiado por la misma institución.

"* Docente investigador de la Facultad de Psicología Universidad Santo Tomás. Correo electrónico: fredyrodriguez@usantotomas.edu.co

"** Docente de la Facultad de Psicología Universidad Santo Tomás. Correo electrónico: carolinavalencia@usantomas.edu.co

***** Estudiante de la Facultad de Psicología Universidad Santo Tomás. Correo electrónico: erikamariagaitan@gmail.com

****** Estudiante de la Facultad de Psicología Universidad Santo Tomás. Correo electrónico: sergio.gonzalezr@usantotomas.edu.co

Estudiante de la Facultad de Psicología Universidad Santo Tomás. Correo electrónico: juanleondiaz@usantotomas.edu.co

Vol. 7, N. ${ }^{\circ}$ y 2 , Enero - diciembre de 2017, pp. 81-102 
estudiantes seleccionados de forma aleatoria, a los cuales se les aplicó una lista de chequeo de hábitos de saludables, la Escala Revisada de Motivos para la Actividad Física y el Cuestionario de Percepción de Barreras para la Práctica de la Actividad Física. Se realizó un análisis descriptivo y posteriormente una regresión logística de la clasificación de activos e insuficientemente activos en relación con los motivos y barreras estudiadas. Los resultados muestran un mayor porcentaje de estudiantes clasificados como activos físicamente frente a otras investigaciones. Se corrobora la falta de motivación intrínseca y de tiempo como barreras más importantes; sin embargo, en cuanto a la predicción de ser clasificado como físicamente activo, el motivo de apariencia física cobra relevancia.

Palabras clave: hábitos saludables, barreras, motivos, actividad física. 


\section{Healthy habits, motives and barriers in the conduct of physical activity in university students}

\section{Abstract}

The main objective of this research was to know the reasons, barriers and healthy habits associated with physical activity in Psychology students at a university in Bogota. The sample consisted of 145 randomly selected students, who were given a checklist of healthy habits, the Revised Scale of Motivation for Physical Activity and the Barriers Perception Questionnaire for Physical Activity Practice; We performed a descriptive analysis and then a logistic regression of the classification of assets and insufficient assets in relation to the reasons and barriers studied. The results show a higher percentage of students as physically active, compared to other research, corroborating the lack of intrinsic motivation and time as more important barriers; However, regarding the prediction of being classified as physically active, the physical appearance motif becomes relevant.

Keywords: Healthy habits, barriers, motives and physical activity. 


\section{Introducción}

Durante décadas, la investigación ha mostrado continuamente que la práctica regular de actividad física (AF) tiene efectos benéficos, tanto físicos como psicológicos, en las personas que la realizan. Para alcanzar dichos efectos positivos, se han desarrollado diversas recomendaciones, entre las que se sugiere que las personas realicen como mínimo de tres sesiones semanales de por lo menos 20 minutos de actividad física moderada, con un día intermedio de descanso (American College of Sports Medicine, 1990). Otra recomendación indica que las personas requieren acumular 30 minutos de actividad con intensidad moderada todos los días (Gauvin, Lévesque y Richard, 2001). Pese a lo anterior, la recomendación utilizada en la mayoría de los contextos proviene de la Organización Mundial de la Salud (2010), la cual expresa que debe ser mayor a 150 minutos semanales con intensidad moderada a vigorosa.

Entre los beneficios físicos de la práctica regular de AF se reporta mejoría en la fuerza y la flexibilidad muscular; facilitación en la disminución de sobrepeso corporal; mejoramiento del funcionamiento del corazón y los pulmones, así como regulación del colesterol y del azúcar en la sangre (Arboleda, 2003). Entre los beneficios psicológicos encontrados se cuenta con el aumento de las percepciones de bienestar, de autoestima y de autoeficacia; incluso se utiliza de manera complementaria al tratamiento de diferentes trastornos mentales (Calfas, Sallis y Nader, 1991; Elavsky et al., 2005; Lee y Rusell, 2003, citados por Saxena, Ommeren, Tang, y Armstrong, 2005; Taylor, Sallis y Needle, 1985).

Sin embargo, a pesar de dichos beneficios, las estimaciones de realización de AF indican que la mayoría de las personas son poco activas. Para el caso de la población joven en el continente americano, alrededor del $73 \%$ no realiza la suficiente AF para obtener beneficios para su salud (National Center for Chronic Disease Prevention and Health Promotion, 2002). Esta situación también se evidencia en la Encuesta Nacional de Salud efectuada en una universidad chilena, donde se encontró la tendencia de exceso de peso y sedentarismo en estudiantes universitarios (Alarcón et al., 2015). En Colombia, Gómez, Lucumi, Girón y Espinosa (2004), encontraron que en algunas localidades de Bogotá el $56.3 \%$ de los adultos jóvenes son inactivos físicamente en su tiempo libre. Específicamente en jóvenes universitarios 
bogotanos se ha encontrado que subutilizan los escenarios dispuestos en su institución para la realización de AF (Sánchez, Torres y Velásquez, 2005), y el nivel de sedentarismo reportado en otra investigación es de 82.4 \% (Jerez, $2005)$, cifra mayor a la reportada para el continente. La excepción a esta tendencia es la encontrada por Rubio y Varela (2016), con tan solo un 25\% de la muestra clasificada con bajo nivel de AF.

En general, la AF es un concepto que se refiere tanto al deporte como al ejercicio físico. La AF alude a cualquier movimiento corporal resultado de músculos esqueléticos y que, por ende, involucra un gasto de energía; mientras el ejercicio físico incluye la AF (Caspersen, Powell y Christenson, 1985), diferenciándose de esta en que implica el movimiento corporal planeado, estructurado y repetitivo realizado con el objeto de mejorar o mantener algún componente de la condición física. Pese a la anterior distinción, la mayor parte de los deportes y las actividades que mejoran la condición física, se consideran ejercicio físico en diferentes contextos, lo cual podría explicar los numerosos instrumentos relacionados con variables psicológicas que utilizan de manera sinónima ejercicio físico y AF. En el caso de la medición, en este trabajo se han seleccionado instrumentos que evalúan tanto el deporte como el ejercicio.

La AF ha sido señalada como una de las conductas que componen un estilo de vida saludable (Saxena et al., 2005; Warburton, Nicol y Bredin, 2006). Igualmente, el Centro de Control y Prevención de Enfermedades (CDC, por sus siglas en inglés), indica que las conductas que hacen parte del estilo de vida contribuyen al menos en un $50 \%$ al estado de salud (Minden y Jason, 2002; Wrotniak, Zimmer, Dingle, Dingle, Miller, Knoell y Weiss, 2007).

Desde la perspectiva de salud, un estilo de vida es conceptualizado como un patrón con carácter estable, visible en un individuo o grupo y que se relaciona con la salud o con la enfermedad (Nutbeam, Aaro y Catford, 1989); a estos patrones también se les denomina hábitos. Belloc y Breslow (1972) refieren un listado de hábitos que inciden positivamente en la calidad de vida de las personas, entre los que se encuentran: dormir entre $7 \mathrm{y}$ 8 horas diarias, desayunar, no fumar, ingerir licor de manera moderada, mantenerse cerca del peso ideal, evitar comer entre comidas y la realización de ejercicio regular. 
Por su parte, la motivación para hacer ejercicio físico y deporte ha sido ampliamente abordada desde diferentes perspectivas teóricas, lo cual ha dado lugar a varias alternativas para la intervención y la medición, ya sea mediante aproximaciones estrictamente hacia el ejercicio o hacia el deporte; incluso se comprenden el ejercicio y el deporte como parte de una sola categoría (Roberts, 1995; Cantón, 1997). Los motivos para ejercitarse han sido considerados por algunos investigadores como la clave para la adherencia, debido a su poder predictivo. En este sentido, los más importantes han sido los denominados motivos intrínsecos, tal como lo confirman los hallazgos efectuados desde la teoría de la autodeterminación de Deci y Ryan (2000).

Desde esta teoría, es necesario partir de necesidades innatas no solo fisiológicas, sino también psicológicas, entre ellas, de relación, de competencia y autonomía. La primera involucra el deseo de conectarse con otros de manera significativa; la segunda relata el deseo de lograr lo que se propone a partir de sus recursos comportamentales; y la tercera explica el deseo de que el ser humano organice su propio comportamiento y experiencias (Celis-Merchan, 2006). De acuerdo con Deci y Ryan (2000), las personas pueden actuar tanto por recompensas externas o evitación de castigo, como por el disfrute mismo de la actividad. Este último tipo de motivación es la que permite mantener de manera más persistente a una persona realizando una actividad, lo que se expresa como el grado más alto de autodeterminación (Celis-Merchan, 2006).

Según algunas investigaciones, hasta el momento se ha logrado sostener que la práctica de ejercicio tiende a ser de corto tiempo cuando se encuentra asociada a motivos extrínsecos, mientras que la asociada con motivos intrínsecos relacionados con un patrón de conducta plenamente autodeterminado predice mayor permanencia (Ntoumanis, 2005) y/o la intención de incrementar la práctica (Biddle, Soos y Chatzisarantis, 1999; Standage, Duda y Ntoumanis, 2003). El sentido de afiliación, que es considerado como extrínseco, podría explotarse en el diseño de los programas, como lo sugieren los resultados obtenidos por Izquierdo-Porrera, Powell, Reiner y Fontaine (2002), quienes encontraron relación entre la afiliación como un motivador para el ejercicio y el gasto calórico semanal en un grupo de 48 mujeres afroamericanas que participaron en una intervención comunitaria en una iglesia. 
Finalmente, se encuentra la variable denominada como barreras, consideradas por algunos autores como razones (Centers for Disease Control and Prevention, s. f.; Weinberg y Gould, 2010). En general, las barreras hacia la AF se clasifican en dos grandes categorías: personales y ambientales. En cuanto a las personales, se encuentran las razones que las personas ofrecen por su inactividad, como la escasez de tiempo, la poca capacidad percibida para la AF, la falta de disfrute con el ejercicio, la ausencia de motivación y la pérdida de apoyo o compañía de las personas que conforman su red de apoyo, los costos que puede involucrar y el temor a la enfermedad o lesión (Perri et al., 2002). Por su parte, las barreras ambientales comprenden factores como la inseguridad, la polución y la falta de transporte (Center for Disease Control and Prevention, 2004). Hasta el momento, existe suficiente investigación que resalta como principal barrera la falta de tiempo (Daskapan, Handan y Eker, 2006; Murray, 2006; Ruiz, García y Díaz, 2007).

El examen conjunto de variables que pueden asociarse a la realización o no de AF ha mostrado diferentes resultados. Para el segmento estudiantil, se han analizado variables psicosociales, teniendo en cuenta su participación en la AF, su intención de realizarla y los beneficios y barreras percibidos. En una investigación, los estudiantes reportaron percibir beneficios sociales y psicológicos en su imagen corporal y en la salud; mientras que respecto a las barreras, mencionaron el tiempo y el esfuerzo (Myers y Roth, 1997). En otra investigación con estudiantes colegiales, la AF fue predicha por su realización en horas de la tarde, su percepción como una actividad divertida y el apoyo recibido de la familia (Sallis, Prochaska, Taylor, Hill y Geraci, 1999).

En población adulta, se evaluó la asociación entre AF y sedentarismo con barreras, disfrute y preferencias. Se encontró que las personas que reportaron gran disfrute y gusto por la AF tenían mayor probabilidad de estar físicamente activos. Las personas que percibieron barreras como clima, costos y otros aspectos personales tenían menor probabilidad de ser físicamente activos. La preferencia por la conducta sedentaria se asoció con la disminución de la probabilidad de estar activo y la percepción del clima como barrera se asoció con el aumento de la probabilidad de no estar activo (Salmon, Owen, Crawford, Bauman y Sallis, 2003).

También se han estudiado las barreras percibidas por adultos trabajadores que cuentan con un centro de acondicionamiento en el lugar de trabajo y se encontró que la percepción de barreras externas ambientales 
cuenta de manera significativa en la decisión de no asistir a dicho centro y en la disminución de la duración de la estadía en el mismo; y que, por otra parte, las barreras internas pesan de manera significativa en la frecuencia de las visitas al mismo (Schwetschenau, O’Brien, Cunningham y Jex, 2008).

En el estudio de Cerin, Vandelanote, Leslie y Merom (2008) se analizaron los factores sociodemográficos, psicosociales y la autoeficacia, en relación con la AF. Se encontraron componentes como: edad, educación, sobrepeso u obesidad, razones para la elección del vecindario, facilidades de las personas residentes en vecindarios para acceder a la recreación, el disfrute y tiempo utilizado en la realización de AF y la autoeficacia.

Teniendo en cuenta el escaso conocimiento de factores relacionados con la actividad física en jóvenes universitarios en el contexto colombiano, el propósito de la presente investigación es identificar los motivos y las barreras que puedan predecir la realización de AF en una población universitaria de la carrera de psicología.

\section{Metodología}

El estudio es de tipo descriptivo-correlacional, ya que se evaluó el grado de relación entre variables. Se trató de establecer cómo se asociaron las variables psicosociales con la realización de AF.

\section{Participantes}

Partiendo de un universo de 488 estudiantes activos, se procedió a seleccionar de manera aleatoria estratificada por semestre a 216 elementos muestrales, con un nivel de confianza del $95 \%$. La muestra final estuvo conformada por 145 estudiantes que aceptaron participar en el estudio.

\section{Instrumentos}

Para la presente investigación se utilizaron tres instrumentos: 
1. Escala Revisada de Motivos para la Actividad Física (MPAM-R), adaptada al español por Celis-Merchán (2006). El nombre del instrumento en inglés es Motives for Physical Activities Measure Revised, y fue desarrollado por Ryan, Frederick, Lepes, Rubio y Sheldon (1997).

2. Lista de Chequeo de Hábitos de Salud, realizada a partir de la lista propuesta por Belloc y Breslow (1972). En la investigación que realizaron estos autores con 7000 personas se identificó que entre más hábitos saludables reportaban las personas menos enfermedades habían tenido y mejor se habían sentido. Los hábitos en la lista de chequeo fueron: dormir entre 7 y 8 horas diarias, desayunar, no fumar, ingerir licor de manera moderada, mantenerse cerca del peso ideal, evitar comer entre comidas y la realización de ejercicio regular. A la lista se añadió un ítem sobre la cantidad de actividad física en el tiempo libre (deporte/ejercicio) que las personas consideraban que realizaban por semana.

3. Barriers to Being Active Quiz (CDC, s. f.), traducido por los autores y validado por tres expertos en psicología de la salud.

\section{Procedimiento}

Se obtuvo el listado de estudiantes inscritos en cada semestre para poder obtener la muestra y seleccionarla de manera aleatoria; posteriormente, durante dos semanas se procedió a pasar por los salones para aplicar el instrumento y, finalmente, se realizó el análisis de datos en el programa SPSS, versión 17.

\section{Resultados}

La muestra estuvo conformada por un $84 \%$ de mujeres y un 16\% de hombres. Los rangos de edad con mayor número de participantes fueron: entre 18 y 20 años, con 78 estudiantes, y entre 21 y 23 años, con 47 estudiantes, mientras que el resto de rangos estuvo por debajo de 12 (figura 1). 
Figura 1. Distribución de participantes por edad

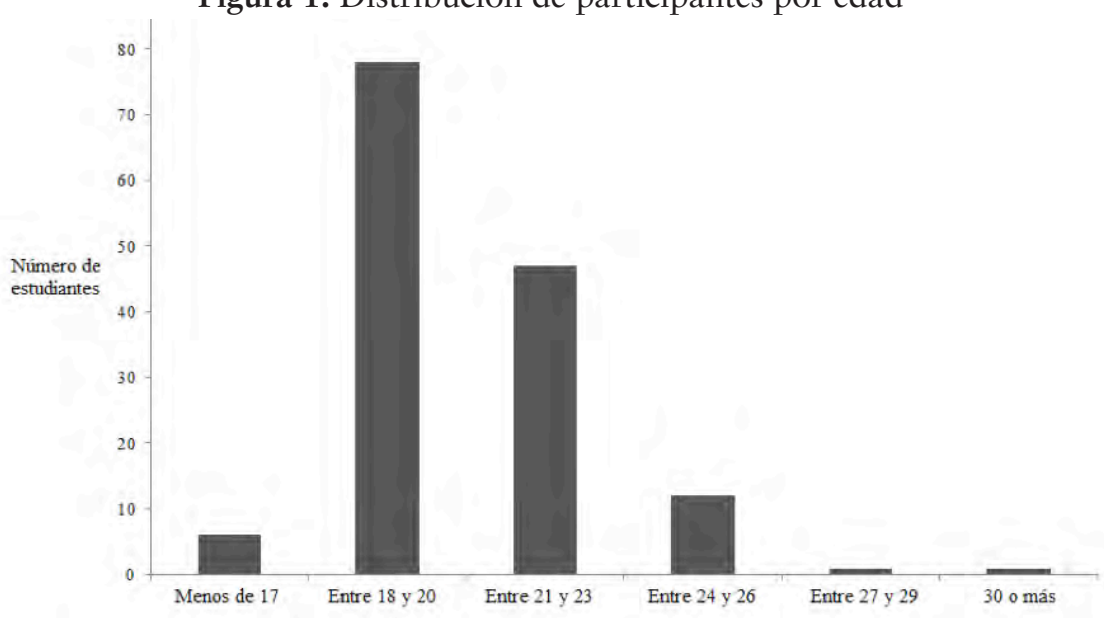

Fuente: elaboración propia.

El estrato socioeconómico mayormente representado es el tres, seguido del cuatro, el dos, el cinco y, finalmente, el uno (figura 2). Aplicando la prueba de inferencia de Chi cuadrado de Pearson para dos muestras independientes, no se encontraron diferencias en la proporción de activos e inactivos, según el estrato (Chi²:3.11; Sig: 0.539>0.05).

Figura 2. Distribución de participantes por estrato socioeconómico

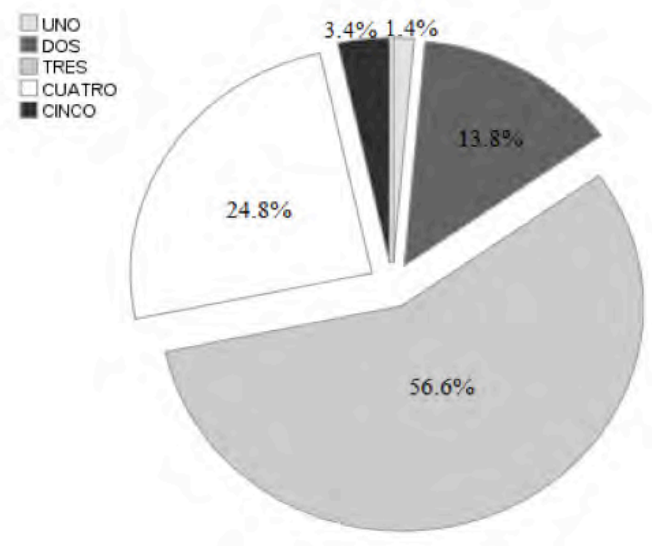

Fuente: elaboración propia. 
El $72 \%$ de los estudiantes manifestó encontrarse en nivelación académica. Por otra parte, los semestres con menor participación en la investigación fueron quinto y séptimo, con 8 personas cada uno, y el que más participación tuvo fue sexto con 22 (figura 3 ).

Figura 3. Distribución de participantes por semestre académico

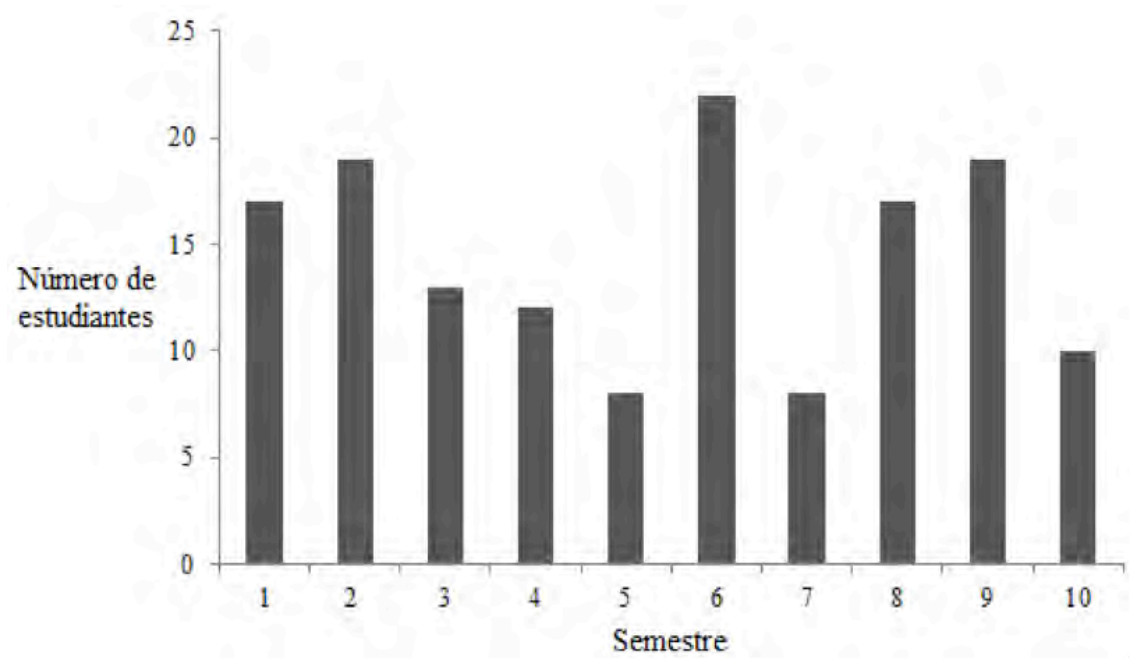

Fuente: elaboración propia.

Respecto a las conductas que hacen parte de un estilo de vida saludable, se identificó que la principalmente reportada es el desayunar, seguida del consumo ocasional de alcohol, mientras que en el último lugar está la realización de actividad física vigorosa (tabla 1 ). 
Tabla 1. Estilos de vida reportados por los participantes

\begin{tabular}{lcc}
\hline \multicolumn{1}{c}{ Conducta } & n & $\%$ \\
\hline Desayunar casi todos los dias & 106 & 73.1 \\
Comer rara vez entre comidas & 41 & 28.3 \\
Estar cerca del peso adecuado & 72 & 49.7 \\
Nunca fumar & 90 & 62.1 \\
Ingerir alcohol de vez en cuando & 97 & 66.9 \\
Realizar actividad fisica vigorosa & 35 & 24.1
\end{tabular}

Fuente: elaboración propia.

Los participantes se dividieron entre activos físicamente (150 minutos o más de actividad física) e insuficientemente activos (menos de 150 minutos) y se halló que el $67.2 \%$ clasifica como activos físicamente. Por otra parte, se identificaron como físicamente activos la mayoría de hombres y mujeres (figura 4), y se estableció que no existen diferencias en la cantidad de activos e insuficientemente activos según el sexo. Esto último se corroboró mediante la prueba de inferencia de Chi cuadrado de Pearson para dos muestras independientes, obteniéndose la significación bilateral del estadístico exacto de Fisher (significación bilateral: 0.495>0.05).

Figura 4. Distribución hombres y mujeres de acuerdo con el nivel de actividad física

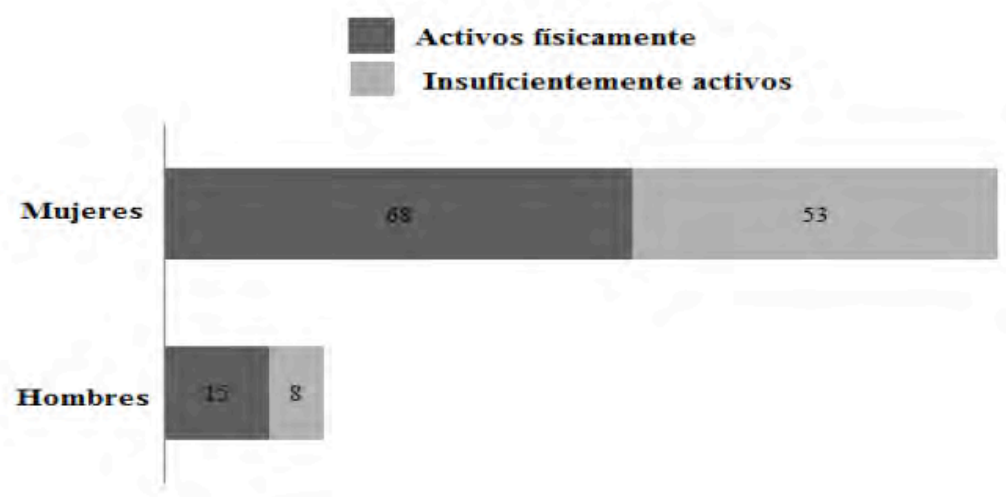

Fuente: elaboración propia. 
Para determinar los motivos percibidos para la realización de actividad física en la muestra, se seleccionaron para cada categoría los puntajes superiores a una desviación por encima de la media. El motivo predominante fue la diversión, seguido por la competencia y la salud. En último lugar se puntuó la apariencia y el aspecto social (tabla 2).

Tabla 2. Motivos para hacer actividad física

\begin{tabular}{lccc}
\hline \multicolumn{1}{c}{ Motivo } & Puntaje de corte & n & \% \\
\hline Diversión & $>=40$ & 29 & 20 \\
Competencia & $>=40$ & 27 & 18.6 \\
Apariencia & $>=33$ & 25 & 17.2 \\
Salud & $>=31$ & 27 & 18.6 \\
Social & $>=22$ & 25 & 17.2 \\
\hline
\end{tabular}

Fuente: elaboración propia.

Respecto a las barreras percibidas para la realización de AF se reportó como más frecuente la falta de tiempo, seguida por la consideración de no realizar la AF con suficiencia por la falta de energía y de las influencias sociales, quedando en el último lugar el temor a las lesiones (tabla 3).

Tabla 3. Barreras hacia la actividad física

\begin{tabular}{lcc}
\hline \multicolumn{1}{c}{ Barrera } & $\mathbf{n}$ & $\mathbf{\%}$ \\
\hline Falta de tiempo & 99 & 73.5 \\
Influencias sociales & 53 & 36.5 \\
Falta de energía & 53 & 36.5 \\
Falta de voluntad & 61 & 42 \\
Temor a las lesiones & 25 & 17.2 \\
Falta de habilidad & 31 & 21.3 \\
Falta de recursos & 40 & 27.5 \\
\hline
\end{tabular}

Fuente: elaboración propia. 
Se compararon las medias respecto a los motivos y las barreras en función del sexo (una vez realizada la prueba de normalidad Kolmogorov Smirnov para cada variable). Mediante la prueba T para muestras independientes se encontraron diferencias entre hombres y mujeres en la percepción de falta de habilidad para la realización de AF (F: 4.27; sig: 0.035; T: 2.32; significancia bilateral: 0.026); en este caso, las mujeres perciben menor habilidad que los hombres.

Una vez revisados los supuestos se ejecutó el análisis de regresión logística binaria: el bloque 0 indica que hay una probabilidad del $58.5 \%$ de acierto en asegurar que un estudiante de psicología es físicamente activo. El incremento en esta probabilidad dependerá, en el modelo, de las variables del número de hábitos saludables y el motivo de apariencia para realizar actividad física.

En el bloque del modelo la puntuación de eficiencia estadística de ROA indicó que se puede mejorar la predicción ( $\mathrm{Chi}^{2}: 4.284$; gl; $\left.\mathrm{p}<0.05\right)$. El valor de $\mathrm{R}^{2}$ de Nagelkerke fue de 0.44 , lo que indica que el modelo propuesto explica el $44 \%$ de la varianza de la variable dependiente. Por otra parte, la prueba de Hosmer y Lemeshow señala que la varianza arrojada por el modelo explica un porcentaje significativo de la varianza de la variable dependiente, pues se acerca bastante a $1\left(\mathrm{Chi}^{2}: 3.140 ; \mathrm{p}=0.925\right)$.

Para el modelo de regresión logística binaria, el bloque 1 indica que hay un $61.5 \%$ de probabilidad de acierto en el resultado de la VD cuando se conoce la cantidad de conductas saludables y si el motivo para ser físicamente activo es la apariencia.

El modelo muestra también que entre mayor sea la relevancia del motivo de apariencia, mayor será la probabilidad de ser físicamente activo (Exp [B]: 1.042). Como último punto de este análisis el valor de Wald revela que este último hallazgo es factible generalizarlo a la población (Wald 4.134; gl $1 ; \mathrm{p}<0.05)$.

\section{Discusión}

Teniendo en cuenta el criterio de 150 minutos semanales de actividad moderada/vigorosa, se encontró que el $67.2 \%$ de los estudiantes podrían clasificarse como activos. Este porcentaje es similar a lo reportado en el 
continente americano (National Center for Chronic Disease Prevention and Health Promotion, 2002) y mayor para lo encontrado en algunas localidades de Bogotá (Gómez, Lucumi, Girón y Espinosa, 2004).

Para el caso específico de la población universitaria, el porcentaje de clasificados como activos físicamente es mayor al reportado en otro estudio colombiano (Jerez, 2005). A la vez, se ve ligado con la investigación de Varela, Duarte, Salazar, Lema y Tamayo (2011), según la cual el $75.3 \%$ de los jóvenes colombianos no practican o pocas veces realizan algún deporte con fines competitivos y, además, se indicaba que el sedentarismo era superior en esta población. En este sentido, el reporte de realización de actividad física desde el enfoque de salud indica que un porcentaje bajo de la muestra realiza actividad física vigorosa. Sin embargo, a pesar de dedicar mayor tiempo a actividades sedentarias, estas personas pueden beneficiarse por la acumulación de la realización de AF moderada varios días a la semana (Gauvin, Lévesque y Richard, 2001).

Es necesario para futuras investigaciones indagar, además de la estimación global del tiempo de realización de actividad física y la percepción de realizarla, la frecuencia, tiempo e intensidad en periodos determinados, como semanas y meses, el horario en el que se realiza habitualmente y la percepción de las condiciones climáticas (Sallis et al., 1999; Salmon et al., 2003). Esto podría lograrse con la medición de la actividad física mediante métodos objetivos (Dugdill y Stratton, 2007), junto con las actividades realizadas en el tiempo libre y de ocio, ya que no se conoce el contexto de práctica de la AF y/o ejercicio y si se está realizando en los horarios académicos, como parte de los espacios académicos de la universidad disponibles para tal fin, o en los tiempos de ocio y tiempo libre (Nuviala, Ruiz y García, 2003).

Respecto a los hallazgos relacionados con el estilo de vida, es necesario aclarar que aunque esté conformado por conductas y hábitos, no implica que las personas tengan comportamientos completamente saludables. Esto es evidente en el alto porcentaje de jóvenes que reportaron el consumo de alcohol, como segundo "hábito saludable"; por lo anterior, se considera necesario indagar con mayor precisión acerca de los patrones y condiciones de consumo en esta población, que permitan el desarrollo de un programa aún más integral. Es importante en este sentido que se estime frecuencia y cantidad de consumo en periodos de tiempo como semana o meses, para 
verificar si esta población presenta consumo moderado o problemático de alcohol.

No se encontró un motivo para la práctica de la AF predominante; sin embargo, se esperaría idealmente que la diversión fuese el más reportado, debido a que pertenece a la motivación intrínseca, la cual facilitaría la adherencia, de acuerdo con la teoría de Deci y Ryan (2000). Entonces, el desarrollo de un programa de AF con miras a la adhesión podría enfocarse en potenciar más este último aspecto, dejando en segundo lugar los otros tipos de motivos. Pese a lo anterior, conociendo el carácter de adhesión que presenta, el sentido de afiliación y el aspecto relacional como motivo deberían explotarse más, como lo hicieron Izquierdo-Porrera et al., (2002), pues la tercera mayor barrera esclarecida fue que los grupos sociales de los estudiantes encuestados no realizan AF. Esta conclusión también encuentra soporte en el trabajo de Pastor, Balaguer y García-Merita (2006), el cual versa sobre las dimensiones del autoconcepto, predictoras de conductas de riesgo para la salud; entre ellas menciona la adecuación de la conducta que se encuentra relacionada con los dominios sociales, como por la percepción de una normativa impuesta, la aceptación de los pares y la competencia siendo un motivador en el deporte. Por su parte, Almagro y Conde (2012) pudieron predecir la intención de continuar siendo físicamente activos en adolescentes jugadores de baloncesto, a partir de un clima motivacional entre los jugadores y el entrenador que implica compromiso, competencia, motivación intrínseca y relaciones con los demás.

La falta de tiempo, la barrera más reportada en la literatura, también lo fue en esta investigación, la cual es interpretada como una excusa personal por algunos autores (Daskapan, Handan y Eker, 2006; Kang, Zhu, Ragan y Frogley, 2007; Murray, 2006; Ruiz, García y Díaz, 2007; Valero, Gómez, Gavala, Ruiz y García, 2007; Weinberg y Gould, 2010), por ser una razón dada para la inactividad o para la realización de actividades sedentarias, que aumenta la probabilidad de no adhesión y de no realización de la conducta.

Además de la percepción de barreras individuales, se requiere indagar acerca de barreras externas ambientales que pueden estar impidiendo la realización de AF (Kang et al., 2007; Schwetschenau et al., 2008) o de posibles barreras psicosociales como la falta de apoyo social (Nuviala et al., 2003).

Teniendo en cuenta las diferencias de género relacionadas con las barreras, se hace necesario que en las actividades diseñadas para mujeres se 
fomente aún más el desarrollo de las habilidades básicas que les permitan obtener una sensación de disfrute suficiente para mantener una motivación intrínseca, para así reducir el riesgo de deserción, el cambio de percepción de barreras a través de cambios personales relacionados con el manejo del tiempo y el aumento del apoyo social para la realización de la misma (Sallis et al., 1999).

Probablemente uno de los datos que más retaría a un futuro programa de promoción en AF sea la barrera que ocupa el segundo lugar, que es la falta de voluntad. Sería ideal buscar la manera de generar, identificar o vincular algún tipo de beneficio de la $\mathrm{AF}$ con un mejor desempeño académico, pues es el factor que más interesa a los estudiantes: algunos estudios indican que no afecta el rendimiento académico de forma negativa y algunos sugieren una correlación positiva (Strong et al., 2005). Por otra parte, sería interesante incluir otras variables de tipo estructural que tienden a predecir el abandono de la actividad física, como lo presentaron Isorna, Ruiz y Rial (2013).

El estudio de la adhesión a la AF es necesario, ya que permite identificar personas que tienen el hábito y pueden percibir beneficios físicos y psicológicos de su realización, como lo muestra el estudio de Arruza, Arribas, Gil De Montes, Irazusta, Romero y Cecchini (2008). En dicho trabajo, el factor que permitió la percepción de beneficios en el estado de ánimo fue la duración de la práctica, factor que no fue tenido en cuenta en esta investigación.

Respecto al género, dado el mayor número de mujeres y el hecho de que no se encontraron diferencias de género en distintos aspectos de la $\mathrm{AF}$ estudiados, no pueden realizarse inferencias sobre la existencia de variables asociadas a esta categoría. Sin embargo, hay que considerar que en el estudio de Romero, Carrasco, Sañudo y Chacón (2010) se encontraron diferencias en hombres en la realización de AF y en la percepción de la salud relacionada, por lo que es necesario para futuras investigaciones contar con muestras representativas de hombres y mujeres que permitan el análisis por género.

Como la mayor parte de la muestra estuvo compuesta por mujeres y esta población presenta una mayor probabilidad de no realizar actividad física y de abandonarla (Dishman y Buckworth, 1997), puede subrayarse la idea de la mejora de la apariencia a través de la realización regular y 
mantenida de AF para favorecer la adhesión, de acuerdo con los hallazgos del modelo de regresión.

Es necesario estudiar también variables de tipo contextual que faciliten o inhiban la AF, como son las prácticas en la institución educativa y la familia. Solamente teniendo una aproximación amplia, podrán desarrollarse intervenciones efectivas que promuevan la $\mathrm{AF}$ de diferentes segmentos poblacionales, tal como lo demandan las posturas contemporáneas relacionadas con la prevención y la promoción.

\section{Referencias}

Alarcón, M., Delgado, P., Caamaño, F., Osorio, A., Rosas, M. y Cea, F. (2015). Estado nutricional, niveles de actividad física y factores de riesgo cardiovascular en estudiantes de la Universidad Santo Tomás. Revista Chilena de Nutrición, 42(1), 70-76. Recuperado de http://www.scielo.cl/scielo.php?script=sci_arttext \&pid=S0717-75182015000100009

Almagro, B. y Conde, C. (2012). Factores motivacionales como predictores de la intención de ser físicamente activos en jóvenes jugadores de baloncesto. Cuadernos de Psicología del Deporte, 12, 1-4.

American College of Sport Medicine (1990). The recommended quantity and quality of exercise for developing and maintaining cardiorespiratory and muscular fitness in healthy adults. Medicine and Science in Sports and Exercise, 22(2), 265-274.

Arboleda, L. (2003). Beneficios del ejercicio. Hacia la Promoción de la Salud, 8. Recuperado de http://promocionsalud.ucaldas.edu.co/downloads/Revista\% 208_8.pdf

Arruza, J., Arribas, S., Gil De Montes, L, Irazusta, S., Romero, S. y Cecchini, J. (2008). Repercusiones de la duración de la actividad físico-deportiva sobre el bienestar psicológico. Revista Internacional de Medicina y Ciencias de la Actividad Física y el Deporte, 8(30), 171-183.

Belloc, N. y Breslow, L. (1972). Relationship of physical health status and health practices. Preventive Medicine, 1, 409-421.

Biddle, S., Soos, I. y Chatzisarantis, N. (1999). Predicting physical activity intentions using goal perspectives and self-determination theory approaches. European Psychologist, 4(2), 83-89. 
Calfas, K., Sallis, J. y Nader, P. (1991). The development of scales to measure knowledge and preference for diet and physical activity behavior in 4 to 8-year-old children. Journal of Developmental and Behavioral Pediatrics, 12, 185-190.

Cantón, E. (1997). Motivación en la actividad física y deportiva. Revista Electrónica de Motivación y Emoción, 3 (5-6). Recuperado de http://reme.uji.es/articulos/ acante1372212100/texto.html.

Caspersen, C., Powell, K. y Christenson, G. (1985). Physical activity, exercise, and physic fitness: definitions, and distinctions for health-related research. Public Health Report, 100(2), 126-131

Celis-Merchan, G. (2006). Adaptación al español de la escala revisada de motivos para la actividad física (MPAM-R) y el cuestionario de clima deportivo (SCQ). Avances en Medición, 4(1), 73-90.

Centers for Disease Control and Prevention. (2004). CDC Releases Guidelines to Promote Physical Activity in Young People. Recuperado de http://www.cdc. gov/media/pressrel/exerci1.htm

Centers for Disease Control and Prevention. (s. f.). Barriers to Being Active Quiz. Recuperado de www.cdc.gov/nccdphp/dnpa/physical/life/barriers_quiz.pdf

Cerin, E., Vandelanote, C., Leslie, E. y Merom, D. (2008). Recreational facilities and leisure-time physical activity: an analysis of moderators and self-efficacy as a mediator. Health Psychology, 27(2 Suppl.), 126-135.

Daskapan, A., Handan, E. y Eker, L. (2006). Perceived barriers to physical activity in university students. Journal of Sports Science and Medicine, 5, 615-620.

Deci, E. y Ryan, R. (2000). Self-determination theory and the facilitation of intrinsic motivation, social development, and well-being. American Psychologist, 55, 68-78.

Dishman, R. K. y Buckworth, J. (1997). Adherence to physical activity. En W. P. Morgan (ed.), Physical Activity and Mental Health (pp. 63-80). Washington: Taylor y Francis.

Dugdill, L. y Stratton, G. (2007). Evaluating Sport and Physical Activity Interventions: A Guide for Practitioners. Salford: Salford University Press.

Elavsky, S., McAuley, E., Motl, R., Konopack, J., Marquez, D., Hu, L., Jerome, G. y Diener, E. (2005). Physical activity enhances long-term quality of life in older adults: Efficacy, esteem, and affective influences. Annals of Behavioral Medicine, 30, 138-145. 
Gauvin, L., Lévesque, L. y Richard, L. (2001). Helping people initiate and maintain a more active lifestyle. A public health framework for physical activity promotion research. En R. Singer, H. Hausenblas y C. Janelly (eds.), Handbook of Sport Psychology (pp. 716-739). New York: Wiley.

Gómez, L., Lucumí, D., Girón, S. y Espinosa, G. (2004). Conglomeración de factores de riesgo de comportamiento asociados a enfermedades crónicas en adultos jóvenes de dos localidades de Bogotá, Colombia: importancia de las diferencias de género. Revista Española de Salud Pública, 78(4), 493-504.

Isorna, M., Ruiz, F. y Rial, A. (2013). Variables predictoras del abandono de la práctica físico-deportiva en adolescentes. Cultura, Ciencia y Deporte, 8(23), 93-102.

Izquierdo-Porrera, A., Powell, C., Reiner, J. y Fontaine, K. (2002). Correlates of exercise adherence in an african american church community. Cultural Diversity and Ethnic Minority Psychology, 6(4), 389-394.

Jerez, A. (29 de mayo de 2005). Médicos lanzan alerta sobre sedentarismo, cigarrillo y alcohol en estudiantes universitarios de hoy. El Tiempo Recuperado de http:// eltiempo.terra.com.co/educ/notieducacion/

Kang, M., Zhu, Z., Ragan, B. G. y Frogley M. (2007). Exercise barrier severity and perseverance of active youth physical disabilities. Rehabilitation Psychology, 52(2), 170-176.

Minden, J. y Jason, L. (2002). Preventing chronic health problems. En L. Jason, y D. Glenwick (eds.), Innovative Strategies for Promoting Health and Mental Health Across the Life Span (pp. 227-243). New York: Springer Publishing Company.

Murray, L. (2006). Sport, exercise and physical activity: Public participation, barriers and attitudes. Research Findings, 20. Recuperado de http://www.scotland.gov.uk/Publications/2006/09/29134926/1

Myers, R. S. y Roth, D. L. (1997). Perceived benefits and barriers to exercise and stage of exercise adoption in young adults. Health Psychology, 16(3), 277-283.

National Center for Chronic Disease Prevention and Health Promotion (2002). Promoting physical activity. American Journal of Preventive Medicine, 22, 73-102.

Ntoumanis, N. (2005). A prospective study of participation in optional school physical education using a self-determination theory framework. Journal of Educational Psychology, 97(3), 444-453. 
Nutbeam, D., Aaro, L. y Carford, J. (1989). Understanding children's health behavior: The implication for health promotion for young people. Social Science and Medicine, 29(3), 317-325.

Nuviala N., A., Ruiz J., F. y García M., M. E. (2003). Tiempo libre, ocio y actividad física en los adolescentes, la influencia de los padres. Retos. Nuevas Tendencias en Educación Física, Deporte y Recreación, 6, 13-20.

Organización Mundial de la Salud (OMS, 2010). Recomendaciones Mundiales sobre Actividad Física para la Salud. Recuperado de http://www.who.int/dietphy/ publications/9789 241599979/es/index.html

Pastor, Y., Balaguer, I. y García-Merita, M. (2006). Relaciones entre el autoconcepto y el estilo de vida saludable en la adolescencia media: un modelo exploratorio. Psicothema, 18(1), 18-24.

Perri, M., Antón, S. Durning, P., Ketterson, T., Sydeman, S., Berlant, N., Kanasky, W., Newton, R., Limacher, M., Martin, A. (2002). Adherence to exercise prescriptions: effects of prescribing moderate versus higher levels of intensity and frequency. Health Psychology, 21(5), 452-458.

Roberts, G. (1995). Motivación en el deporte y el ejercicio. Bilbao: Desclée de Brouwer.

Romero, S., Carrasco, L., Sañudo, B. y Chacón, F. (2010). Actividad física y percepción del estado de salud en adultos sevillanos. Revista Internacional de Medicina y Ciencias de la Actividad Física y el Deporte, 10(39), 380-392.

Rubio, R. y Varela, M. (2016) Barreras percibidas en jóvenes universitarios para realizar actividad física. Revista Cubana de Salud Pública, 42(1), 61-69. Recuperado de http://www.scielosp.org/pdf/rcsp/v42n1/spu07116.pdf

Ruiz, F., García, M. y Díaz, A. (2007). Análisis de las motivaciones de práctica de actividad física y de abandono deportivo en la ciudad de La Habana (Cuba). Anales de Psicología, 1(23), 152-166.

Ryan, R., Frederick, C., Lepes, D., Rubio, N. y Sheldon, K. (1997). Intrinsic motivation and exercise adherence. Sport Psychology, 28, 335-354.

Sallis, J. M., Prochaska, J. J., Taylor, W. C., Hill, J. O. y Geraci, J. C. (1999). Correlates of physical activity in a national sample of girls and boys in grades 4 through 12. Health Psychology, 18(4), 410-415.

Salmon, J., Owen, N., Crawford, D., Bauman, A. y Sallis, J. F. (2003). Physical activity and sedentary behavior: a population-based study of barriers, enjoyment, and preference. Health Psychology, 22(2), 178-188. 
Sánchez, H., Torres, D. y Velásquez, M. (2005). Análisis de factores psicológicos que inciden en la no práctica, práctica y mantenimiento de la actividad física en estudiantes de la Universidad Nacional de Colombia. Revista Colombiana de Psicología, 14, 137-147.

Saxena, S., Ommeren, M., Tang, K. y Armstrong, T. (2005). Mental health benefits of physical activity. Journal of Mental Health. 14(5), 445-451.

Schwetschenau, H. M., O’Brien, W. H., Cunningham, C. J. L. y Jex, S. M. (2008). Barriers to physical activity in an on-site corporate fitness center. Journal of Occupational Health Psychology, 13(4), 371-380.

Standage, M., Duda, J. y Ntoumanis, N. (2003). A model of contextual motivation in physical education: using constructs from self-determination and achievement goal theories to predict physical activity intentions. Journal of Educational Psychology, 95(1), 97-110.

Strong, W., Malina, R., Blimkie, A., Daniels, S., Dishman, R., Bernard, G., Hergenroeder, A., Nixon, P., Pivarnik, J., Rowland, T., Stewart, T. y Trudeau, F. (2005). Evidence based physical activity for school-age youth. The Journal of Pediatrics, 8, 732-737.

Taylor, C., Sallis, J y Needle, R. (1985). The relation of physical activity and exercise to mental health. Public health reports, 100(2), 195-202.

Valero V., A., Gómez L., M., Gavala G., J., Ruiz J., F. y García M., M. E. (2007). ¿Por qué no se realiza actividad físico-deportiva en el tiempo libre? Motivos y correlatos sociodemográficos. Retos. Nuevas Tendencias en Educación Física, Deporte y Recreación, 12, 13-17.

Varela, M., Duarte, C., Salazar, I., Lema, L., y Tamayo, J. (2011). Actividad física y sedentarismo en jóvenes universitarios de Colombia: prácticas, motivos y recursos para realizarlas. Colombia Médica, 42(3), 269-277. Recuperado de http://www.bioline.org.br/request?rc11049

Warburton, D., Nicol, C. y Bredin. (2006). Health benefits of physical activity: the evidence. Canadian Medical Association 174(6), 801-809.

Weinberg, R. y Gould, D. (2010). Fundamentos de Psicología del Deporte y del Ejercicio Físico. Madrid: Panamericana.

Wrotniak, B., Zimmer, N., Dingle, K., Dingle, A., Miller, A., Knoell, A. y Weiss, E. (2007). Physical activity, health, and dietary patterns of middle school children. Pediatric Physical Therapy, 19(3), 203-10. 